\title{
Comparative effectiveness of combined antiplatelet treatments in acute minor ischaemic stroke
}

\begin{abstract}
Shina Kim, ${ }^{1}$ Joon-Tae Kim (D) , ${ }^{1}$ Ji Sung Lee, ${ }^{2}$ Beom Joon Kim (D) , ${ }^{3}$ Jong-Moo Park, ${ }^{4}$ Kyusik Kang, ${ }^{5}$ Soo Joo Lee, ${ }^{6}$ Jae Guk Kim, ${ }^{6}$ Jae-Kwan Cha, ${ }^{7}$ Dae-Hyun Kim, ${ }^{7}$ Tai Hwan Park, ${ }^{8}$ Sang-Soon Park, ${ }^{8}$ Kyung Bok Lee, ${ }^{9}$ Jun Lee, ${ }^{10}$ Keun-Sik Hong, ${ }^{11}$ Yong-Jin Cho, ${ }^{11}$ Hong-Kyun Park, ${ }^{11}$ Byung-Chul Lee, ${ }^{12}$ Kyung-Ho Yu, ${ }^{12}$ Mi Sun Oh, ${ }^{12}$ Dong-Eog Kim, ${ }^{13}$ Wi-Sun Ryu, ${ }^{13}$ Jay Chol Choi, ${ }^{14}$ Jee-Hyun Kwon, ${ }^{15}$ Wook-Joo Kim, ${ }^{15}$ Dong-Ick Shin, ${ }^{16}$ Sung-II Sohn, ${ }^{17}$ Jeong-Ho Hong (D) , ${ }^{17}$ Man-Seok Park, ${ }^{1}$ Kang-Ho Choi, ${ }^{1}$ Ki-Hyun Cho, ${ }^{1}$ Juneyoung Lee, ${ }^{18}$ Hee-Joon $\mathrm{Bae}^{3}$
\end{abstract}

To cite: Kim S, Kim J-T, Lee JS, et al. Comparative effectiveness of combined antiplatelet treatments in acute minor ischaemic stroke. Stroke \& Vascular Neurology 2021;0. doi:10.1136/svn-2020-000841

- Additional supplemental material is published online only. To view, please visit the journal online (http://dx.doi.org/10. 1136/svn-2020-000841).

Received 29 December 2020 Accepted 27 May 2021

Check for updates

(c) Author(s) (or their employer(s)) 2021. Re-use permitted under CC BY-NC. No commercial re-use. See rights and permissions. Published by BMJ.

For numbered affiliations see end of article.

Correspondence to

Dr Joon-Tae Kim;

alldelight2@jnu.ac.kr

Dr Hee-Joon Bae; braindocbae@gmail.com

\section{ABSTRACT}

Background No study has thoroughly compared the effectiveness of combined antiplatelet treatments (other than clopidogrel-aspirin) versus clopidogrel-aspirin or aspirin alone for early secondary prevention in acute ischaemic stroke.

Methods We identified patients with acute, minor, noncardiogenic ischaemic stroke treated with aspirin alone, clopidogrel-aspirin or other combination treatment. Propensity scores considering the inverse probability of treatment weighting were used to adjust for baseline imbalances. The primary outcome was the composite of all strokes (ischaemic or haemorrhagic), myocardial infarction and all-cause mortality at 3 months.

Results Among 12234 patients (male: $61.9 \%$; age: $65.5 \pm 13$ years) who met the eligibility criteria, aspirin, clopidogrelaspirin and other combination treatments were administered in $52.2 \%, 42.9 \%$ and $4.9 \%$ of patients, respectively. In the crude analysis, the primary outcome event at 3 months occurred in $14.5 \%$ of the other combination group, $14.4 \%$ of the aspirin group and $13.0 \%$ of the clopidogrel-aspirin group. In the weighted Cox proportional hazards analysis, the 3-month primary outcome event occurred less frequently in the clopidogrel-aspirin group than in the other combination group (weighted HR: 0.82 (0.59-1.13)), while no association was found between the aspirin group (weighted HR: 1.04 (0.76-1.44)) or other combination group and the 3-month primary outcome.

Conclusion Other combined antiplatelet treatment, compared with aspirin alone or clopidogrel-aspirin, was not associated with reduced risks of primary composite vascular events or recurrent stroke during the first 3 months after stroke. Therefore, the results suggest that other combination treatments, particularly the cilostazolbased combination, may not be effective alternatives for clopidogrel-aspirin to prevent early vascular events in patients with acute minor stroke. Further exploration in clinical trials will be needed.

\section{INTRODUCTION}

Because the risk of recurrent vascular events and potential benefits of secondary prevention strategies are highest early after ischaemic stroke, separate strategies for short-term and long-term secondary prevention have been developed. ${ }^{1}$ The early initiation of aspirin within 48 hours of stroke onset is primarily recommended for early secondary prevention in patients with acute, non-cardiogenic ischaemic stroke, reducing the risk of recurrent ischaemic stroke by $23 \% .^{2}$ In patients with minor non-cardioembolic ischaemic stroke, dual antiplatelet treatment with clopidogrel-aspirin within 12-24 hours of onset is more effective than aspirin alone in reducing the risk of recurrent ischaemic stroke at 3 months ${ }^{34}$; this therapy regimen is currently recommended for early secondary prevention in patients with acute minor stroke or highrisk transient ischaemic attack (TIA). ${ }^{5}$

The efficacy of other combination treatments (other than clopidogrel-aspirin), however, is controversial and has not been well established considering early secondary prevention in acute ishaemic stroke (AIS). Rothwell et al found that combined treatment with aspirin-dipyridamole was not more effective than aspirin for early secondary prevention within 12 weeks of onset. ${ }^{6}$ In the Triple Antiplatelets for Reducing Dependency after Ischaemic Stroke study, ${ }^{7}$ combined treatment with aspirin-clopidogrel-dipyridamole was not more effective than clopidogrel monotherapy or combined aspirin-dipyridamole therapy in preventing recurrent stroke and even increased the bleeding risk. By contrast, combined treatment with cilostazol-aspirin was superior to clopidogrel monotherapy or aspirin monotherapy in patients with ischaemic stroke with presumed arterial origin ${ }^{8}$; however, this study included only patients 
with subacute ischemic stroke. The effectiveness of other combination treatments versus clopidogrel-aspirin or aspirin has not yet been thoroughly investigated.

Therefore, we investigated whether other combination antiplatelet treatments, compared with clopidogrelaspirin or aspirin alone, could reduce the risk of early vascular events in patients with acute minor ischaemic stroke.

\section{METHODS}

\section{Study subjects}

This study was a retrospective analysis of data from the Clinical Research Collaboration for Stroke-Korea (CRCS-K) registry, a prospective and web-based registry of consecutive patients with acute stroke or TIA admitted to 16 academic hospitals or regional stroke centres in South Korea (http://www.stroke-crc.or.kr/ecrf). Detailed information about the CRCS-K registry has been published previously. ${ }^{910}$ In the current study, 56853 patients with stroke or TIA who were admitted between January 2011 and July 2018 were initially screened. We included patients with acute minor ischaemic stroke (within 24 hours of onset), defined as those with a National Institutes of Health Stroke Scale (NIHSS) score of 5 or less. Those with cardioembolic stroke indicating a need for anticoagulation were excluded. In this study, patients treated with aspirin alone or combined antiplatelets, such as clopidogrel-aspirin, were finally included. The detailed eligibility criteria and patient selection flow chart are shown in online supplemental figure 1 .

\section{Data collection}

The data collection process has been described in previous studies. ${ }^{9} 10$ Briefly, the demographic, clinical, imaging and laboratory data were prospectively collected. The baseline data, including NIHSS scores, were collected for all the patients, and the stroke subtypes were classified according to the Trial of Org 10172 in Acute Stroke Treatment (TOAST) criteria after complete diagnostic profiling. ${ }^{112}$ The following data were directly obtained from the registry database: (1) demographics, including age, sex and body mass index; (2) medical history, including previous TIA, previous stroke, previous coronary artery disease $(\mathrm{CAD})$, previous peripheral artery disease, hypertension, diabetes mellitus, dyslipidaemia, smoking (current, recent and ex-smoker) and atrial fibrillation; (3) medications, including previous antiplatelet medications, previous antihypertensive medications, previous antidiabetic treatments, previous lipid-lowering medications other than statins and previous statin medications; (4) stroke characteristics and acute treatments, including the onset-to-arrival time, initial NIHSS score, prestroke modified Rankin Scale score, ischaemic stroke subtype according to the TOAST criteria and relevant arterial steno-occlusive disease (large artery stenosis/ occlusion, LASO), which was categorised as no stenosis, mild stenosis $(<50 \%)$, moderate-to-severe stenosis $(\geq 50 \%)$ and total occlusion; (5) laboratory data, including white blood cell counts, creatinine serum levels, glucose at presentation, platelet counts, fasting low-density lipoprotein cholesterol and systolic blood pressure; and (5) in-hospital and discharge treatments, including antihypertensives, statins, lipid-lowering treatments other than statins and antidiabetic therapies. For continuous variables, if less than $5 \%$ of the values were missing, the data were imputed with the median values. The study subjects were divided into three groups for comparison according to the antiplatelet regimens used during hospitalisation: aspirin, clopidogrel-aspirin and other combination therapy. The profiles of the detailed antiplatelet regimens and protocols are listed in online supplemental table 1.

\section{Outcomes}

The primary outcome was a composite of all types of stroke (ischaemic and haemorrhagic), myocardial infarction (MI) and all-cause mortality during the first 3 months after the index stroke. The secondary outcomes were the following individual events: (1) all types of stroke (ischaemic and haemorrhagic), (2) MI, (3) allcause mortality, (4) ischaemic stroke and (5) haemorrhagic stroke. Stroke events included both progressive and recurrent stroke, as in the Platelet-Oriented Inhibition in New TIA and Minor Ischaemic Stroke trial. ${ }^{13}$ The detailed definitions of the outcome events used in the current study have been described in a previous report. ${ }^{9}$

Vascular events were prospectively captured during hospitalisation and the 3-month follow-up period by dedicated stroke nurses or physicians based on predefined protocols at routine clinic visits or by telephone interviews. To ensure the accuracy of the outcomes and minimise inter-interviewer discrepancies, a uniformly structured questionnaire was used by trained personnel. ${ }^{9}$

\section{Statistical analysis}

The details of the statistical analysis are described in the online supplemental methods. Briefly, to minimise confounding and residual selection bias in observational treatment comparisons, a propensity score weighting method was applied to control for imbalances in various baseline characteristics across the three groups. In this study, a generalised propensity score was applied using multinomial logistic regression, and the corresponding inverse probabilities of treatment weights (the reciprocals of the propensity scores) were estimated. Survival curves were constructed using adjusted Kaplan-Meier estimates and compared using inverse probability of treatment weighting (IPTW) log-rank tests. ${ }^{14}$ For sensitivity analysis, cilostazol-based combination treatments, initial NIHSS scores of 0-3, first-ever stroke, and study periods between 2014 and 2018 were analysed. An absolute standardised difference of $<0.1$ for each baseline covariate was assumed as a minimal and acceptable imbalance among the three groups. All the reported $\mathrm{p}$ values were two-sided, and a $\mathrm{p}$ value $<0.05$ was considered to indicate statistical significance. All the analyses were performed using SAS V.9.4. 
Table 1 General characteristics of the subjects

\begin{tabular}{|c|c|c|c|c|c|c|}
\hline & $\begin{array}{l}\text { Aspirin } \\
\text { monotherapy }\end{array}$ & Clopidogrel-aspirin & $\begin{array}{l}\text { Other } \\
\text { combination }\end{array}$ & $P$ valuet & $\mathbf{P 1}^{*}$ & $\mathbf{P 2}^{*}$ \\
\hline $\mathrm{N}$ & 6391 & 5243 & 600 & & & \\
\hline Age, years $($ mean $\pm S D)$ & $64.3 \pm 13.4$ & $66.7 \pm 12.5$ & $68.1 \pm 11.4$ & $<0.001$ & $<0.001$ & 0.018 \\
\hline Male, n (\%) & $3938(61.6)$ & $3281(62.6)$ & $350(58.3)$ & 0.107 & 0.228 & 0.085 \\
\hline Onset-to-admission time, $\mathrm{n}(\%)$ & & & & 0.104 & 0.868 & 1.000 \\
\hline Within 12 hours & $4710(73.7)$ & $3953(75.4)$ & $451(75.2)$ & & & \\
\hline $12-24$ hours & $1681(26.3)$ & $1290(24.6)$ & $149(24.8)$ & & & \\
\hline Body mass index (mean $\pm S D)$ & $23.9 \pm 3.3$ & $23.9 \pm 3.2$ & $24.1 \pm 3.5$ & 0.543 & 0.398 & 0.344 \\
\hline NIHSS score, median (IQR) & $2(1-3)$ & $2(1-4)$ & $2(1-3)$ & $<0.001$ & 0.002 & 0.738 \\
\hline $0, n(\%)$ & $1349(21.1)$ & $997(19.0)$ & $107(17.8)$ & & & \\
\hline 1 & $1363(21.3)$ & $1075(20.5)$ & $116(19.3)$ & & & \\
\hline 2 & $1391(21.8)$ & $1055(20.1)$ & $116(19.3)$ & & & \\
\hline 3 & $1009(15.8)$ & $793(15.1)$ & $114(19.0)$ & & & \\
\hline 4 & $768(12.0)$ & $730(13.9)$ & $91(15.2)$ & & & \\
\hline 5 & $511(8.0)$ & $593(11.3)$ & $56(9.3)$ & & & \\
\hline TOAST, n (\%) & & & & $<0.001$ & $<0.001$ & 1.000 \\
\hline LAA & 2332 (36.5) & 2605 (49.7) & $298(49.7)$ & & & \\
\hline SVO & 2311 (36.2) & $1447(27.6)$ & $175(29.2)$ & & & \\
\hline $\mathrm{OE}$ & $204(3.2)$ & $176(3.4)$ & $16(2.7)$ & & & \\
\hline UD & $1544(24.2)$ & 1015 (19.4) & $111(18.5)$ & & & \\
\hline History of TIA, n (\%) & $94(1.5)$ & 168 (3.2) & $34(5.7)$ & $<0.001$ & $<0.001$ & 0.004 \\
\hline History of stroke & $639(10.0)$ & $1038(19.8)$ & $253(42.2)$ & $<0.001$ & $<0.001$ & $<0.001$ \\
\hline History of PAD & $14(0.2)$ & $27(0.5)$ & $6(1.0)$ & 0.002 & 0.011 & 0.288 \\
\hline History of CAD & $250(3.9)$ & $490(9.3)$ & $85(14.2)$ & $<0.001$ & $<0.001$ & $<0.001$ \\
\hline Hypertension & 3865 (60.5) & $3603(68.7)$ & $452(75.3)$ & $<0.001$ & $<0.001$ & 0.002 \\
\hline Diabetes mellitus & $1870(29.3)$ & $1786(34.1)$ & $269(44.8)$ & $<0.001$ & $<0.001$ & $<0.001$ \\
\hline Dyslipidaemia & $1761(27.6)$ & $1820(34.7)$ & $244(40.7)$ & $<0.001$ & $<0.001$ & 0.008 \\
\hline Smoking status & & & & $<0.001$ & $<0.001$ & 0.017 \\
\hline Never smoker & 3641 (57.0) & 3093 (59.0) & $372(62.0)$ & & & \\
\hline Ex-smoker, $>5$ years & $504(7.9)$ & $480(9.2)$ & $58(9.7)$ & & & \\
\hline Recent smoker, within 5 years. & $321(5.0)$ & $252(4.8)$ & $41(6.8)$ & & & \\
\hline Current smoker & $1925(30.1)$ & $1418(27.0)$ & $129(21.5)$ & & & \\
\hline Atrial fibrillation & $41(0.6)$ & $25(0.5)$ & $8(1.3)$ & 0.032 & 0.134 & 0.032 \\
\hline Prior antiplatelet use & $893(14.0)$ & $1742(33.2)$ & $354(59.0)$ & $<0.001$ & $<0.001$ & $<0.001$ \\
\hline Prior antihypertensive use & $2625(41.1)$ & $2727(52.0)$ & $376(62.7)$ & $<0.001$ & $<0.001$ & $<0.001$ \\
\hline Prior statin use & 759 (11.9) & $1182(22.5)$ & $215(35.8)$ & $<0.001$ & $<0.001$ & $<0.001$ \\
\hline Prior lipid-lowering agent use & $101(1.6)$ & $120(2.3)$ & $18(3.0)$ & 0.004 & 0.020 & 0.554 \\
\hline Prior antidiabetic use & $1314(20.6)$ & $1370(26.1)$ & $215(35.8)$ & $<0.001$ & $<0.001$ & $<0.001$ \\
\hline Multiple lesions & $515(8.1)$ & $667(12.7)$ & $78(13.0)$ & $<0.001$ & $<0.001$ & 1.000 \\
\hline LASO & & & & $<0.001$ & $<0.001$ & 0.155 \\
\hline No stenosis & $4155(65.0)$ & $2693(51.4)$ & $295(49.2)$ & & & \\
\hline Mild (<50\%) & 525 (8.2) & $581(11.1)$ & $81(13.5)$ & & & \\
\hline Significant $(\geq 50 \%)$ & $992(15.5)$ & $1200(22.9)$ & $151(25.2)$ & & & \\
\hline Occlusion & 719 (11.3) & 769 (14.7) & 73 (12.2) & & & \\
\hline In-hospital treatment & & & & & & \\
\hline
\end{tabular}


Table 1 Continued

\begin{tabular}{|c|c|c|c|c|c|c|}
\hline & $\begin{array}{l}\text { Aspirin } \\
\text { monotherapy }\end{array}$ & Clopidogrel-aspirin & $\begin{array}{l}\text { Other } \\
\text { combination }\end{array}$ & $P$ value $†$ & $\mathbf{P 1}^{*}$ & P2* \\
\hline Antidiabetics & $1413(22.1)$ & $1360(25.9)$ & $208(34.7)$ & $<0.001$ & $<0.001$ & $<0.001$ \\
\hline Anti-lipid-lowering agents & $3713(58.1)$ & $3520(67.1)$ & $431(71.8)$ & $<0.001$ & $<0.001$ & 0.040 \\
\hline Statins & $5800(90.8)$ & 4885 (93.2) & $538(89.7)$ & $<0.001$ & 0.764 & 0.003 \\
\hline WBC count $\left(\times 10^{3} / \mu \mathrm{L}\right)$ & $8.0 \pm 2.8$ & $8.0 \pm 2.8$ & $8.0 \pm 3.3$ & 0.777 & 0.587 & 0.616 \\
\hline Creatinine (mg/dL) & $0.93 \pm 0.85$ & $1.03 \pm 0.98$ & $1.12 \pm 1.17$ & $<0.001$ & $<0.001$ & 0.059 \\
\hline Haemoglobin (g/dL) & $13.9 \pm 1.9$ & $13.8 \pm 1.8$ & $13.4 \pm 1.8$ & $<0.001$ & $<0.001$ & $<0.001$ \\
\hline Platelet count $\left(10^{3} / \mu \mathrm{l}\right)$ & $233.8 \pm 65.9$ & $236.3 \pm 68.9$ & $229.9 \pm 71.9$ & 0.027 & 0.236 & 0.039 \\
\hline LDL cholesterol (mg/dL) & $114.8 \pm 35.6$ & $108.5 \pm 36.7$ & $99.9 \pm 38.6$ & $<0.001$ & $<0.001$ & $<0.001$ \\
\hline
\end{tabular}

P1: aspirin monotherapy vs other combination treatment; P2: clopidogrel-aspirin vs other combination treatment.

*Adjusted $\mathrm{p}$ value by Pearson's $\chi^{2}$ test and Fisher's exact test with the Bonferroni adjustment method, the Dwass, Steel, Critchlow-Fligner multiple comparison method or Dunnett's multiple comparison method.

†P value by ANOVA, Kruskal-Wallis test and $\chi^{2}$ test.

CAD, coronary artery disease; LAA, large artery atherosclerosis; LASO, large artery stenosis/occlusion; LDL, low-density lipoprotein; NIHSS, National Institutes of Health Stroke Scale; OE, other aetiology; PAD, peripheral artery diseases; SVO, small vessel occlusion; TIA, transient ischaemic attack; TOAST, Trials of Org 10172 in Acute Stroke Treatment; UD, undetermined aetiology; WBC, white blood cells.

\section{RESULTS}

\section{General characteristics}

After the enrollment process (online supplemental figure 1), 12234 patients (male: $61.9 \%$; age: $65.5 \pm 13$ years) were finally analysed in this study. The median NIHSS score was 2 (IQR: 1-3). The proportions of antiplatelet treatment were as follows: aspirin, 52.2\%; clopidogrelaspirin, 42.9\%; and other combination treatments, $4.9 \%$. The detailed antiplatelet regimens are shown in online supplemental table 1 . Combined treatment with cilostazol-aspirin (67.2\%) was most frequent among the other combination treatments. The general characteristics of the patients are shown in table 1 . The other combination treatment group was the most likely to have risk factors, including a history of stroke, TIA, CAD, hypertension, diabetes mellitus, dyslipidaemia and smoking, and to be on antiplatelet agents, antihypertensives, antidiabetics and statins at stroke onset among the three groups. After IPTW, the distributions of the baseline characteristics were fairly well balanced; the absolute standardised

Table 2 Weighted 3-month event rates after multiple treatment propensity scoring

\begin{tabular}{|c|c|c|c|c|c|c|}
\hline & Aspirin monotherapy & Clopidogrel-aspirin & Other combination & P value* & P1† & P2† \\
\hline \multicolumn{7}{|l|}{ Primary outcome } \\
\hline $\begin{array}{l}\text { Composite of all } \\
\text { strokes, MI and all- } \\
\text { cause mortality }\end{array}$ & 16.28 (15.28-17.29) & $13.04(12.08-14.00)$ & 15.39 (11.33-19.45) & $<0.001$ & 1.000 & 0.406 \\
\hline \multicolumn{7}{|l|}{ Secondary outcome } \\
\hline $\mathrm{Ml}$ & $0.24(0.10-0.38)$ & $0.32(0.15-0.49)$ & $0.00(0.00-0.00)$ & 0.549 & 0.546 & 0.662 \\
\hline Ischaemic stroke & $14.88(13.91-15.85)$ & 11.90 (10.98-12.83) & $15.22(11.18-19.27)$ & $<0.001$ & 1.000 & 0.151 \\
\hline $\begin{array}{l}\text { Haemorrhagic } \\
\text { stroke }\end{array}$ & $0.27(0.12-0.42)$ & $0.28(0.12-0.44)$ & $0.04(0.00-0.30)$ & 0.715 & 0.819 & 0.989 \\
\hline
\end{tabular}

P1: aspirin monotherapy vs other combination; P2: clopidogrel-aspirin vs other combination.

${ }^{*} P$ value by the IPTW log-rank test.

†Adjusted $\mathrm{p}$ value by the Bonferroni adjustment method.

IPTW, inverse probability of treatment weighting; MI, myocardial infarction. 
Table 3 Associations of treatments with the 3-month event outcomes

\begin{tabular}{|c|c|c|c|c|c|c|}
\hline & Crude HR $(95 \% \mathrm{Cl})$ & $P$ value & $\begin{array}{l}\text { Adjusted HR } \\
(95 \% \mathrm{Cl})\end{array}$ & $P$ value & $\begin{array}{l}\text { IPTW*, weighted } \\
\text { HR }(95 \% \mathrm{Cl})\end{array}$ & $P$ value \\
\hline \multicolumn{7}{|l|}{ Primary outcome } \\
\hline Other combination & 1 (ref.) & & 1 (ref.) & & 1 (ref.) & \\
\hline Aspirin monotherapy & 0.98 (0.79 to 1.22$)$ & 0.8814 & 1.17 (0.93 to 1.47$)$ & 0.1659 & 1.05 (0.76 to 1.44$)$ & 0.7762 \\
\hline Clopidogrel-aspirin & 0.88 (0.71 to 1.10$)$ & 0.2741 & 0.92 (0.73 to 1.15$)$ & 0.4543 & 0.82 (0.59 to 1.13$)$ & 0.2211 \\
\hline \multicolumn{7}{|l|}{ All stroke } \\
\hline Other combination & 1 (ref). & & 1 (ref.) & & 1 (ref.) & \\
\hline Aspirin monotherapy & 0.94 (0.75 to 1.17$)$ & 0.5574 & 1.11 (0.88 to 1.40$)$ & 0.3730 & 0.98 (0.71 to 1.36$)$ & 0.9126 \\
\hline Clopidogrel-aspirin & 0.84 (0.68 to 1.06$)$ & 0.1383 & 0.87 (0.69 to 1.09$)$ & 0.2331 & 0.77 (0.56 to 1.06$)$ & 0.1127 \\
\hline \multicolumn{7}{|l|}{ All-cause mortality } \\
\hline Other combination & 1 (ref.) & & 1 (ref.) & & 1 (ref.) & \\
\hline Aspirin monotherapy & 2.85 (0.90 to 8.98$)$ & 0.0744 & $3.93(1.22$ to 12.66$)$ & 0.0218 & 10.66 (2.79 to 40.67$)$ & 0.0005 \\
\hline Clopidogrel-aspirin & 2.51 (0.79 to 7.98$)$ & 0.1184 & 2.87 (0.89 to 9.23 ) & 0.0776 & 7.88 (2.06 to 30.16$)$ & 0.0026 \\
\hline \multicolumn{7}{|l|}{ MI† } \\
\hline Other combination & 1 (ref.) & & & & & \\
\hline Aspirin monotherapy & 1.94 (0.10 to 36.13$)$ & 0.6574 & Non-estimable & & Non-estimable & \\
\hline Clopidogrel-aspirin & 3.23 (0.18 to 59.18$)$ & 0.4286 & Non-estimable & & Non-estimable & \\
\hline \multicolumn{7}{|l|}{ Ischaemic stroke } \\
\hline Other combination & 1 (ref.) & & 1 (ref.) & & 1 (ref.) & \\
\hline Aspirin monotherapy & $0.93(0.75$ to 1.16$)$ & 0.5344 & 1.10 (0.87 to 1.39 ) & 0.4156 & 0.97 (0.70 to 1.34$)$ & 0.8401 \\
\hline Clopidogrel-aspirin & 0.84 (0.67 to 1.05$)$ & 0.1225 & 0.86 (0.69 to 1.09$)$ & 0.2067 & 0.76 (0.55 to 1.05$)$ & 0.0914 \\
\hline \multicolumn{7}{|l|}{ Haemorrhagic stroke } \\
\hline Other combination & 1 (ref.) & & & & & \\
\hline Aspirin monotherapy & 0.89 (0.16 to 5.01$)$ & 0.8960 & Non-estimable & & Non-estimable & \\
\hline Clopidogrel-aspirin & 1.01 (0.18 to 5.69$)$ & 0.9942 & Non-estimable & & Non-estimable & \\
\hline
\end{tabular}

Adjusted variables: age; male sex; initial NIHSS score; arrival time; TOAST classification; history of TIA, stroke, CAD and PAD; HTN; DM; dyslipidaemia; smoking; atrial fibrillation; prior antiplatelet, antihypertensive, antidiabetic, statin or lipid-lowering agent use; multiple lesions; LASO; discharge medications of antihypertensives, antidiabetics, statins and lipid-lowering agents; creatinine; haemoglobin; platelet count; LDL cholesterol; glucose; and SBP.

*Weighted Cox proportional hazards model with robust standard errors.

†Cox PHs regression using Firth's penalised maximum likelihood method.

CAD, coronary artery disease; DM, diabetes mellitus; HTN, hypertension; IPTW, inverse probability of treatment weighting; LASO, large artery stenosis/occlusion; LDL, low-density lipoprotein; NIHSS, National Institutes of Health Stroke Scale; PAD, peripheral artery disease; PHs, proportional hazards; SBP, systolic blood pressure; TIA, transient ischaemic attack; TOAST, Trial of Org 10172 in Acute Stroke Treatment.

differences after IPTW were within a margin of 0.1 for most of the covariates (online supplemental table 2).

\section{Outcomes}

The mean follow-up duration was $93.6 \pm 17.6$ days, with $98.6 \%$ of patients completing 3 months of follow-up. The primary composite outcome of all types of stroke (ischaemic or haemorrhagic), MI and all-cause mortality occurred in 1728 patients, and the 3-month cumulative event rate was $14.3 \%$. For individual outcomes, the 3-month cumulative event rates were $13.3 \%$ for all types of stroke and $1.6 \%$ for all-cause mortality. MI occurred in $0.2 \%$ of patients.

In the crude analysis, the primary outcome event at 3 months occurred in $14.5 \%$ of the other combination group, $14.4 \%$ of the aspirin group and $13.0 \%$ of the clopidogrel-aspirin group (online supplemental table 3). The 3-month unadjusted risks for all types of stroke were significantly lower in the clopidogrel-aspirin group than in the other combination group $(12.5 \%$ vs $14.7 \%$, respectively; $\mathrm{p}=0.049$ ), but they were not different between the aspirin group and other combination group ( $13.6 \%$ vs $14.7 \%$, respectively; $\mathrm{p}=0.56)$. The rate of allcause mortality was lowest in the other combination group compared with the other treatment groups $(0.60 \%$ vs $1.67 \%$ vs $1.50 \%$ for the other combination group vs aspirin group vs clopidogrel-aspirin group, respectively).

In the propensity analysis with IPTW, the primary outcome event and all types of stroke at 3 months were lower in the clopidogrel-aspirin group than in the other combination treatment group $(12.6 \%$ vs $15.2 \%$ for the 
A Composite events of stroke, MI, and all-cause mortality
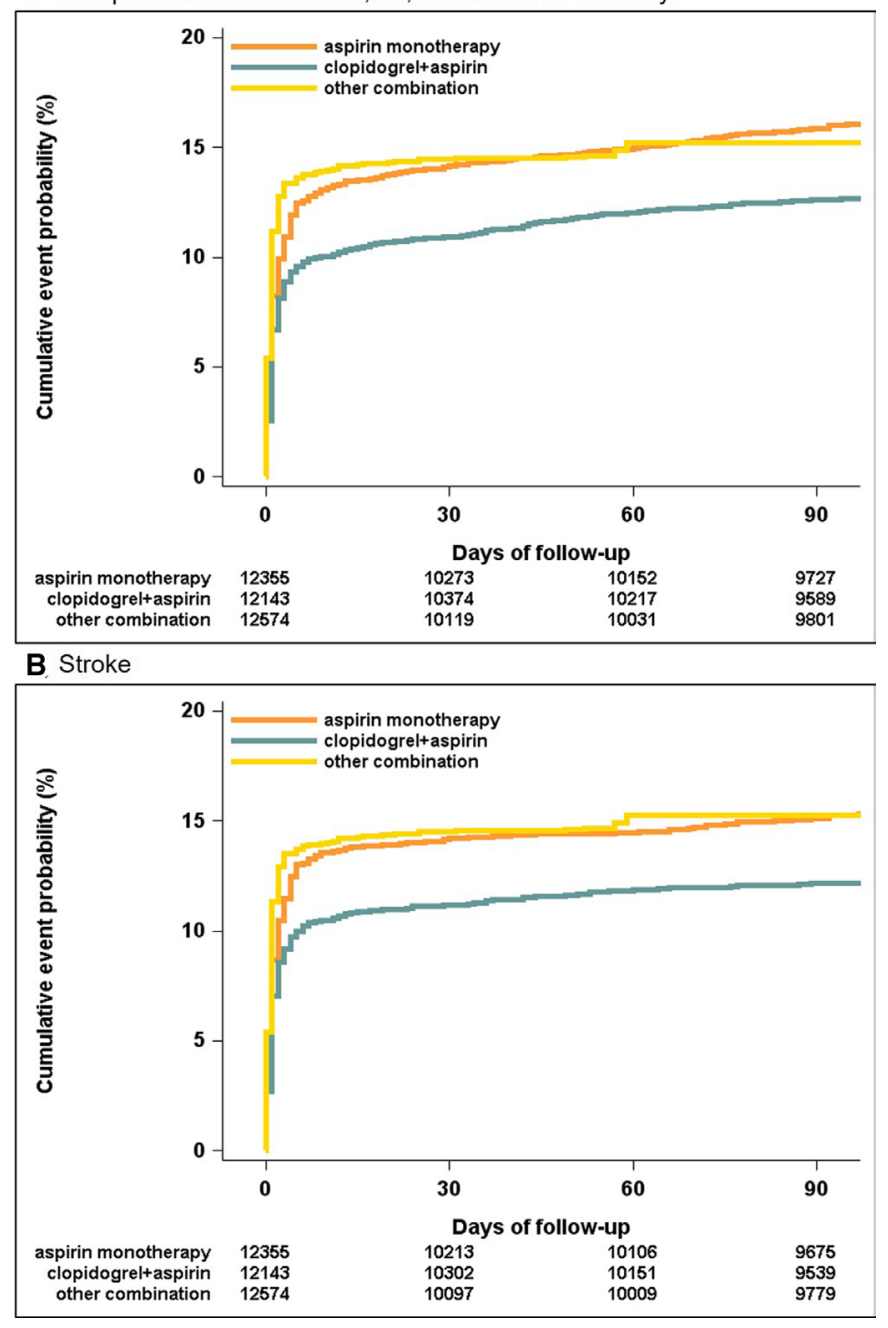

Figure 1 Weighted Kaplan-Meier incidence plots for the primary outcome $(A)$ and all strokes $(B)$ in patients treated with other combined antiplatelets, clopidogrel-aspirin and aspirin monotherapy.

primary outcome and $12.2 \%$ vs $15.3 \%$ for all strokes, respectively), with weighted absolute risk differences of $2.6 \%$ and $3.1 \%$, respectively (table 2). However, no differences were observed between the aspirin group and other combination treatment group $(15.9 \%$ vs $15.2 \%$ for the primary outcome and $15.1 \%$ vs $15.3 \%$ for all strokes, respectively).

In the weighted Cox proportional hazards analysis, no significant associations of the clopidogrel-aspirin versus other combination and aspirin versus other combination groups with the 3-month primary outcome and 3-month all types of stroke were observed. However, compared with other combination treatments, clopidogrel-aspirin and aspirin were associated with a greater risk of all-cause mortality (weighted HR: 7.88 (2.06-30.16) and 10.66 (2.79-40.67), respectively) at 3 months (table 3 ).

Weighted Kaplan-Meier cumulative incidence plots of the primary outcome event and all types of stroke (figure 1A,B) showed that the clopidogrel-aspirin group had the lowest event rates, while the other two groups had similar event rates; all-cause mortality and MI occurred least frequently in the other combination group (online supplemental figure $2 a, b)$.

In the sensitivity analysis, cilostazol-based combination treatment $(\mathrm{n}=547)$ was compared with aspirin alone and clopidogrel-aspirin (online supplemental table 4). Additional sensitivity analyses for the NIHSS score of $0-3$, first-ever stroke and periods between 2014 and 2018 are shown in online supplemental tables 5-10. Results similar to those obtained in the main analyses were observed (table 4 and online supplemental tables 5-10).

\section{DISCUSSION}

In this nationwide, multicentre registry-based analysis of over 12000 patients with acute minor, non-cardiogenic ischaemic stroke, we found that combined antiplatelet treatment other than clopidogrel-aspirin did not reduce the risk of 3-month vascular events but was associated with a reduced risk of all-cause mortality compared with aspirin alone and clopidogrel-aspirin. These results suggest that other combined antiplatelet treatments, mostly cilostazolbased combinations, may have limitations for early secondary prevention in acute minor ischaemic stroke.

Our study found that approximately $5 \%$ of patients with acute minor ischaemic stroke were treated with other combined antiplatelets. Interestingly, cilostazolbased combination therapy was most commonly administered in the study population $(91.2 \%$, most frequently cilostazol-aspirin). Because extended-release dipyridamole is not commercially available for ischaemic stroke in South Korea, physicians considered cilostazol as an alternative for extended-release dipyridamole to replace aspirin or clopidogrel. We also found that other combination treatments were commonly used in patients with high burdens of vascular diseases, traditional stroke risk factors and prior medication for risk factor control. In particular, approximately half of the patients treated with other combined antiplatelets had a history of stroke or TIA and prior antiplatelet use. Therefore, although rigorous adjustment for propensity was performed, our results should be interpreted with caution. However, it is noteworthy to show the real-world practices regarding the characteristics of acute minor stroke patients treated with other combined antiplatelets in a multicentre registry.

Our results provide important information regarding the effectiveness of other combined antiplatelet treatments early after ischaemic stroke. The weighted composite vascular event rate at 3 months was not significantly different between the other combination treatment and aspirin groups $(15.2 \%$ and $15.9 \%$, respectively), but the rate in the clopidogrelaspirin group was lower than that in the other combination treatment group ( $12.6 \%$ vs $15.2 \%$, respectively). Our results seemed to be consistent with the results of a previous metaanalysis. ${ }^{6}$ A meta-analysis of pooled data from randomised trials found that combined treatment with aspirin-dipyridamole did not reduce the risk of early recurrent stroke (within 12 weeks) in patients with acute minor stroke. 
Table 4 Sensitivity analysis for patients treated with the cilostazol-based combination

\begin{tabular}{|c|c|c|c|c|c|c|}
\hline & Crude HR (95\% Cl) & $P$ value & $\begin{array}{l}\text { Adjusted HR } \\
(95 \% \mathrm{Cl})\end{array}$ & $P$ value & $\begin{array}{l}\text { IPTW, }{ }^{*} \text { weighted HR } \\
(95 \% \mathrm{Cl})\end{array}$ & $P$ value \\
\hline \multicolumn{7}{|l|}{ Primary outcome } \\
\hline Aspirin monotherapy & 0.97 (0.77 to 1.22$)$ & 0.79 & 1.14 (0.90 to 1.44$)$ & 0.27 & 0.99 (0.71 to 1.39$)$ & 0.97 \\
\hline Clopidogrel-aspirin & 0.87 (0.69 to 1.10$)$ & 0.24 & 0.89 (0.71 to 1.13$)$ & 0.34 & 0.78 (0.56 to 1.08$)$ & 0.14 \\
\hline Aspirin monotherapy & 0.91 (0.73 to 1.15$)$ & 0.44 & 1.08 (0.85 to 1.36$)$ & 0.55 & 0.93 (0.67 to 1.30$)$ & 0.67 \\
\hline Clopidogrel-aspirin & 0.82 (0.65 to 1.04$)$ & 0.10 & 0.84 (0.67 to 1.07$)$ & 0.16 & 0.73 (0.52 to 1.02$)$ & 0.07 \\
\hline \multicolumn{7}{|l|}{ All-cause mortality } \\
\hline Cilostazol-based combination & 1 (ref.) & & 1 (ref.) & & 1 (ref.) & \\
\hline Cilostazol-based combination & 1 (ref.) & & & & & \\
\hline Aspirin monotherapy & 1.75 (0.09 to 32.65$)$ & 0.71 & Non-estimable & & Non-estimable & \\
\hline Clopidogrel-aspirin & $2.92(0.16$ to 53.48$)$ & 0.47 & Non-estimable & & Non-estimable & \\
\hline
\end{tabular}

Adjusted variables: age; male sex; initial NIHSS score; arrival time; TOAST classification; history of TIA, stroke, CAD and PAD; HTN; DM; dyslipidaemia; smoking; atrial fibrillation; prior antiplatelet, antihypertensive, antidiabetic, statin or lipid-lowering agent use; multiple lesions; LASO; discharge medications of antihypertensives, antidiabetics, statins and lipid-lowering agents; creatinine; haemoglobin; platelet count; LDL cholesterol; glucose; and SBP.

*Weighted Cox proportional hazards model with robust standard errors. †Cox proportional hazards regression using Firth's penalised maximum likelihood method.

CAD, coronary artery disease; DM, diabetes mellitus; HTN, hypertension; IPTW, inverse probability of treatment weighting; LASO, large artery stenosis/occlusion; LDL, low-density lipoprotein; LDL, low-density lipoprotein; NIHSS, National Institutes of Health Stroke Scale; PAD, peripheral artery disease; TIA, transient ischemic attack; TOAST, Trial of Org 10172 in Acute Stroke Treatment.

More importantly, most of the benefit of urgent treatment in previous studies was due to early aspirin use. Additionally, the Cilostazol Stroke Prevention Study for Antiplatelet Combination (CSPS.com) study showed that the efficacy outcomes were generally the same between aspirin monotherapy and dual therapy with cilostazol-aspirin during the initial 3 months. ${ }^{8}$ Because aspirin was administered in most patients treated with other combinations, why no differences were observed in the 3-month primary composite event and all-stroke events between other combination treatments versus aspirin alone can be partly explained.

Nonetheless, all-cause mortality as a main safety outcome was significantly lower in the other combination treatment group than in the aspirin alone or clopidogrel-aspirin group (weighted all-cause mortality rates; $0.18 \%$ vs $1.82 \%$ vs $1.37 \%$, respectively). In previous studies, cilostazol did not increase but tended to reduce the risk of major or life-threatening bleeding compared with aspirin, and adding cilostazol to aspirin seemed to reduce potential life-threatening bleeding compared with single antiplatelet treatment. ${ }^{815} 16$ However, because we could not investigate extracranial bleeding events and the incidence of all-cause mortality was very low in the other combination group, these conclusions should be interpreted with caution. Further study is warranted to confirm our results.

Additionally, the clopidogrel-aspirin group tended to decrease the risks of primary composite vascular events (weighted HR: 0.82 [0.59-1.13]) and all stroke events (weighted HR: 0.77 [0.56-1.06]) compared with other combination treatment groups. Therefore, our results still support the current guidelines for clopidogrel-aspirin, which is recommended as the first choice for early secondary prevention in patients with acute minor ischaemic stroke and high-risk TIA. ${ }^{5}$ However, the results of our study address the necessity of future studies to identify an alternative to clopidogrel-aspirin. A recent trial demonstrated the better efficacy of ticagrelor-aspirin than aspirin alone in early prevention of recurrent stroke, and the findings of this trial still support the effects of a more intensive combined antiplatelet strategy on acute minor stroke. ${ }^{17}$ However, further study is warranted to compare the efficacy of clopidogrelaspirin versus ticagrelor-aspirin for early secondary prevention in acute minor stroke.

This study has several limitations. First, it was a registrybased study with treatment selection arising from clinician decision-making rather than random allocation. Although adjusted for clinically relevant variables, the possibility of residual confounding persists. Second, it was a broad, 
national study, but the patient cohort was restricted to an Asian (South Korean) population; studies involving other racial-ethnic groups are needed to confirm generalisability. Similarly, to date, most clinical trials of cilostazol in patients with ischaemic stroke have been conducted in Asian countries. Third, structured follow-up interviews were not designed to reliably identify all possible adverse bleeding events associated with antithrombotic therapy. Because bleeding is an important issue for combination antiplatelet therapy in AIS, further study is warranted. Fourth, the proportion of other combined treatments was low, possibly leading to statistical bias. To reduce the statistical bias, the IPTW methods were considered. Additionally, other combination treatment groups might be heterogeneous, although a cilostazol (with aspirin) based combination was the most common (over 90\%) among regimens in this group. Fifth, because of the retrospective design, individual information was not available on antiplatelet treatments, such as the duration of treatment, onset-to-treatment time, initial dosage of different treatments and daily dosage of the two treatments. Compliance with antithrombotic therapy regimens was not ascertained by pill counts or direct interviews. Finally, the 3-month vascular event rates were quite high compared with recent publications with similar stroke populations. Because our registry was based on patients with stroke admitted to university hospitals or regional stroke centres, the study subjects might have a relatively higher risk for vascular events other than stroke recurrence compared with the general stroke population, possibly explaining the unusual higher event rates for primary vascular events. However, this study was noteworthy because we thoroughly explored a large population with acute minor ischaemic stroke from a prospective multicentre stroke registry. Thus, these results expanded the understanding of antithrombotic strategies in minor acute stroke.

\section{CONCLUSION}

In this study, for patients with acute, minor, noncardioembolic ischaemic stroke, clopidogrel-aspirin, compared with the early initiation of other combined antiplatelets (mostly cilostazol-based combinations), reduced the risks of primary composite vascular events and recurrent stroke during the first 3 months after stroke. Thus, the results suggest that other combination treatments (other than clopidogrel-aspirin) may not be more effective alternatives for clopidogrel-aspirin to prevent early vascular events in patients with acute minor stroke. Further exploration in clinical trials will be needed.

\footnotetext{
Author affiliations

${ }^{1}$ Department of Neurology, Chonnam National University Hospital, Chonnam National University Medical School, Gwangju, Korea

${ }^{2}$ Clinical Research Center, Asan Institute for Life Sciences, Asan Medical Center, University of Ulsan College of Medicine, Seoul, Korea

${ }^{3}$ Department of Neurology, Cerebrovascular Center, Seoul National University Bundang Hospital, Seongnam, Korea

${ }^{4}$ Department of Neurology, Uijeongbu Eulji Medical Center, Eulji University School of Medicine, Uijeongbu-si, Korea
}

${ }^{5}$ Department of Neurology, Nowon Eulji Medical Center, Eulji University, Seoul, Korea ${ }^{6}$ Department of Neurology, Eulji University Hospital, Eulji University, Daejeon, Korea ${ }^{7}$ Department of Neurology, Dong-a University, Busan, Korea

${ }^{8}$ Department of Neurology, Seoul Medical Center, Seoul, Korea

${ }^{9}$ Department of Neurology, Soonchunhyang University Hospital, Seoul, Korea

${ }^{10}$ Department of Neurology, Yeungnam University Hospital, Daegu, Korea

${ }^{11}$ Department of Neurology, Inje University Ilsan Paik Hospital, Inje University College of Medicine, Goyang, Korea

${ }^{12}$ Department of Neurology, Hallym University Sacred Heart Hospital, Anyang, Korea

${ }^{13}$ Department of Neurology, Dongguk University Ilsan Hospital, Goyang, Korea

${ }^{14}$ Department of Neurology, Jeju National University Hospital, Jeju National

University School of Medicine, Jeju, Korea

${ }^{15}$ Department of Neurology, University of Ulsan College of Medicine, Ulsan, Korea

${ }^{16}$ Department of Neurology, Chungbuk National University Hospital, Cheongju, Korea

${ }^{17}$ Department of Neurology, Keimyung University Dongsan Medical Center, Daegu,

Korea

${ }^{18}$ Department of Biostatistics, Korea University College of Medicine, Seoul, Korea

Contributors Study concept and design: J-TK, SK and H-JB. Acquisition of data: J-TK, M-SP, K-HC, SK, BJK, THP, S-SP, KBL, B-CL, K-HY, MSO, J-KC, D-HK, JL, SJL, JGK, J-MP, KK, Y-JC, K-SH, H-KP, JCC, S-IS, J-HH, D-EK, W-SR, D-IS, W-JK, J-HK and H-JB. Analysis and interpretation of data: J-TK, SK, JSL and H-JB. Drafting of the manuscript: J-TK and SK. Critical revision of the manuscript for important intellectual content: J-TK, SK, M-SP, K-HChoi, K-HCho, BJK, THP, S-SP, KBL, B-CL, K-HY, MSO, J-KC, D-HK, JL, SJL, JGK, J-MP, KK, Y-JC, K-SH, H-KP, JCC, S-IS, J-HH, D-EK, W-SR, D-IS, W-JK, J-HK and H-JB. Statistical analysis: J-TK, JSL and JL.

Funding This research was supported by funding (2020ER620200\#) from Research of Korea Centers for Disease Control and Prevention and the Chong Kun Dang Pharmaceutical Corp. (Seoul, Korea).

Competing interests None declared.

Patient consent for publication Not required.

Ethics approval This study was approved from the local institutional review boards of all the participating centres, including Chonnam National University Hospital and Seoul National University Bundang Hospital. Clinical information was obtained from the CRCS-K registry database. The CRCS-K registry has been collecting clinical information to monitor and improve the quality of stroke care since 2008, with the approval of the institutional review boards of all participating centres. A waiver for informed consent was provided because of study subject anonymity and minimal risk to the participants. The use of the registry database and supplemental review of medical records in this study were approved by the appropriate local institutional review boards. The CRCS-K research committee will provide the data, analytic methods and study materials to other researchers on reasonable request.

Provenance and peer review Not commissioned; externally peer reviewed.

Data availability statement Data are available upon reasonable request. The CRCS-K research committee will provide the data, analytic methods and study materials to other researchers upon reasonable request.

Open access This is an open access article distributed in accordance with the Creative Commons Attribution Non Commercial (CC BY-NC 4.0) license, which permits others to distribute, remix, adapt, build upon this work non-commercially, and license their derivative works on different terms, provided the original work is properly cited, appropriate credit is given, any changes made indicated, and the use is non-commercial. See: http://creativecommons.org/licenses/by-nc/4.0/.

\section{ORCID iDs}

Joon-Tae Kim http://orcid.org/0000-0003-4028-8339

Beom Joon Kim http://orcid.org/0000-0002-2719-3012

Jeong-Ho Hong http://orcid.org/0000-0002-8235-9855

\section{REFERENCES}

1 Hankey GJ. Secondary stroke prevention. Lancet Neurol 2014:13:178-94.

2 Sandercock PAG, Counsell C, Gubitz GJ, et al. Antiplatelet therapy for acute ischaemic stroke. Cochrane Database Syst Rev 2008:CD000029.

3 Wang $Y$, Wang $Y$, Zhao $X$, et al. Clopidogrel with aspirin in acute minor stroke or transient ischemic attack. N Engl J Med 2013;369:11-19. 
4 Johnston SC, Easton JD, Farrant M, et al. Clopidogrel and aspirin in acute ischemic stroke and high-risk TIA. N Engl J Med 2018;379:215-25.

5 Powers WJ, Rabinstein AA, Ackerson T, et al. 2018 guidelines for the early management of patients with acute ischemic stroke: a guideline for healthcare professionals from the American heart Association/ American stroke association. Stroke 2018;49:e46-110.

6 Rothwell PM, Algra A, Chen Z, et al. Effects of aspirin on risk and severity of early recurrent stroke after transient ischaemic attack and ischaemic stroke: time-course analysis of randomised trials. Lancet 2016;388:365-75.

7 Bath PM, Woodhouse LJ, Appleton JP, et al. Antiplatelet therapy with aspirin, clopidogrel, and dipyridamole versus clopidogrel alone or aspirin and dipyridamole in patients with acute cerebral ischaemia (tardis): a randomised, open-label, phase 3 superiority trial. Lancet 2018;391:850-9.

8 Toyoda K, Uchiyama S, Yamaguchi T, et al. Dual antiplatelet therapy using cilostazol for secondary prevention in patients with high-risk ischaemic stroke in Japan: a multicentre, open-label, randomised controlled trial. Lancet Neurol 2019;18:539-48.

9 Kim BJ, Park J-M, Kang K, et al. Case characteristics, hyperacute treatment, and outcome information from the clinical research center for stroke-fifth division registry in South Korea. J Stroke 2015;17:38-53.
10 Kim BJ, Han M-K, Park TH, et al. Current status of acute stroke management in Korea: a report on a multicenter, comprehensive acute stroke Registry. Int J Stroke 2014;9:514-8.

11 Adams HP, Bendixen BH, Kappelle LJ, et al. Classification of subtype of acute ischemic stroke. definitions for use in a multicenter clinical trial. TOAST. trial of ORG 10172 in acute stroke treatment. Stroke 1993;24:35-41.

12 Ko Y, Lee S, Chung J-W, et al. MRI-based algorithm for acute ischemic stroke subtype classification. J Stroke 2014;16:161-72.

13 Johnston SC, Easton JD, Farrant M, et al. Platelet-oriented inhibition in new TIA and minor ischemic stroke (point) trial: rationale and design. Int J Stroke 2013;8:479-83.

14 Xie J, Liu C. Adjusted Kaplan-Meier estimator and log-rank test with inverse probability of treatment weighting for survival data. Stat Med 2005;24:3089-110.

15 Shinohara Y, Katayama Y, Uchiyama S, et al. Cilostazol for prevention of secondary stroke (CSPS 2): an aspirin-controlled, double-blind, randomised non-inferiority trial. Lancet Neurol 2010;9:959-68.

$16 \mathrm{Kim}$ BJ, Lee E-J, Kwon SU, et al. Prevention of cardiovascular events in Asian patients with ischaemic stroke at high risk of cerebral haemorrhage (PICASSO): a multicentre, randomised controlled trial. Lancet Neurol 2018;17:509-18.

17 Johnston SC, Amarenco P, Denison H, et al. Ticagrelor and aspirin or aspirin alone in acute ischemic stroke or TIA. N Engl J Med 2020;383:207-17. 